\title{
Sleep and Health-An Introduction
}

\author{
Sheilja Singh ${ }^{1}$, Namrata Yadav ${ }^{2}$, Sanket Jain ${ }^{3}$
}

\begin{abstract}
Sleep is essential for the normal functioning of a human being. It is a restorative phenomenon. Hypothalamus and ventral periaqueductal gray along with circadian and homeostatic signals regulate the sleep cycle. Sleep architecture is divided into non-rapid eye movement (NREM) and rapid eye movement (REM) phases. Not only theoretically but even in clinical practice, it is evident that sleep deprivation has mild-to-severe effects on every system. Obstructive sleep apnea (OSA) is the most common sleep disorder seen by chest physicians, ear-nose-throat (ENT) surgeons, cardiologists, and general physicians. OSA is gaining insight of surgeons and physicians for preoperative clinical evaluation, and it is mandatory in suspected individuals. Sleep hygiene is helpful in OSA and other sleep-disordered breathing.

Keywords: Sleep architecture, Sleep deprivation, Sleep hygiene.

International Journal of Head and Neck Surgery (2019): 10.5005/jp-journals-10001-1361
\end{abstract}

"A feeling of pleasure envelops your brain. Your day drifts away without sound. Sleep takes your mind to those far away plains, Releasing your feet from the ground."

-Josie Whitehead

Sleep is defined behaviorally as an unconscious state with characteristic supine sleep posture, lack of mobility, closed eyes and increased arousal threshold. Sleep occurs in all the organisms with glial or neuronal network. Sleep is a restorative phenomenon, necessary for recovery and stabilization of synaptic processes. Sleep has always caught the attention right from the ancient times. In the Iliad an ancient greek epic poem, Homer said, "Sleep and death are twin brother". William Shakespeare referred to sleep as "chief nourisher in life fest" and "balm of mind". Likewise, Hippocrates around 400 BC said, "Both sleep and wakefulness when immoderate are bad". Krueger and colleagues elaborated that the neural activity occurring in night hours in sleep protects neural cells that were not used during the day. Although the exact reason why we sleep has not been clear as yet but animals and humans both requiring sleep, makes it important enough for life. There have been many theories to understand functions of sleep in human health.

\section{Functions of Sleep}

- Energy conservation: Minimum oxygen consumption and energy expenditure occurs during sleep leading to accumulation of more adenosine triphosphates (ATPs) in the brain. Sleep affects total body growth by releasing essential hormones like growth hormones.

- Sound sleep has shown to enhance memory, immune system and life survival.

- Glymphatic function of sleep: Sleep allows for an enhanced convective flow from brain to circulation which helps in flushing the soluble neurotoxic waste products like $\beta$-amyloids and others from the brain. This drainage system is functional predominantly in sleep only.

With ageing from new born to elderly age group, there is variation in sleep pattern. Neonates of 38-48 weeks sleep about 16 hours per day and have more REM sleep than adults. Interestingly, sleep is distributed over the whole day, i.e. both day and night. As
1Department of Internal Medicine, Hinduja Khar Surgicals, Mumbai, Maharashtra, India

${ }^{2,3}$ Department of Respiratory Diseases, Lilavati Hospital and Research Center, Mumbai, Maharashtra, India

Corresponding Author: Sheilja Singh, Department of Internal Medicine, Hinduja Khar Surgicals, Mumbai, Maharashtra, India, email: dr_sheilja@yahoo.co.in

How to cite this article: Singh S, Yadav N, et al. Sleep and Health-An Introduction. Int J Head Neck Surg 2019;10(1):1-3.

Source of support: Nil

Conflict of interest: None

Table 1: Sleep architecture

\begin{tabular}{ll}
\hline Sleep stage & $\begin{array}{l}\text { Percentage of total sleep time } \\
(\text { TST) }\end{array}$ \\
\hline NREM 1 & $5-10 \%$ \\
NREM 2 & $50-60 \%$ \\
NREM $3 / 4$ & $15-30 \%$ \\
REM & $20 \%$ \\
\hline
\end{tabular}

age advances, REM sleep decreases. Interestingly, total amount of sleep for an elderly person over 24 hours stays same as that for an young adult. However, sleep architecture gets altered in such a way that stay for long in lighter phases of sleep, have increased sleep latency and frequent fragmentation of sleep. ${ }^{1}$ Elderly people suffer more with sleep disorders and circadian rhythm disorder.

\section{Neurobiology of Sleep}

Sleep is divided in different stages as depicted in Table 1. Ventrolateral preoptic nucleus (VLPO) of the anterior hypothalamus acts as the sleep switch. During sleep, it becomes active and uses GABA and galanin to initiate sleep by inhibiting the regions of the brain which lead to arousal. Besides, some neurons (hypocretin in this nucleus) help stabilize the switch. On the contrary, neurons in the lateral and posterior hypothalamus are the sole source of the awake-promoting neuropeptides hypocretin 1 (Hcrt 1) or orexin A and hypocretin 2 (Hcrt 2) or orexin B. ${ }^{2,3}$ It would be worthwhile to note that when these neurons are lost, narcolepsy can result. Additionally, the

() The Author(s). 2019 Open Access This article is distributed under the terms of the Creative Commons Attribution 4.0 International License (https://creativecommons. org/licenses/by-nc/4.0/), which permits unrestricted use, distribution, and non-commercial reproduction in any medium, provided you give appropriate credit to the original author(s) and the source, provide a link to the Creative Commons license, and indicate if changes were made. The Creative Commons Public Domain Dedication waiver (http://creativecommons.org/publicdomain/zero/1.0/) applies to the data made available in this article, unless otherwise stated. 
Sleep and Health—An Introduction

Table 2: III effects of sleep deprivation

\begin{tabular}{ll}
\hline Central nervous system & Difficulty in concentration, excessive daytime sleepiness, poor coordination skills, mental abilities, \\
mood swings, depression, paranoia \\
Increased risk of ischemic events, hypertension, arrhythmias and even failure \\
Respiratory system & $\begin{array}{l}\text { Pulmonary hypertension, increased frequency of respiratory infections and worsening chronic } \\
\text { respiratory diseases } \\
\text { Immunity }\end{array}$ \\
Sleep deprivation decreases cytokines and makes one more prone to recurrent respiratory infections. \\
It also reduces the killer T-cells \\
- Testosterone production needs at least three hours of uninterrupted sleep, which is about the time \\
of the first REM episode. Waking up throughout the night could affect hormone production \\
- Reduced growth hormone production which is detrimental for child growth \\
- Imbalance between leptin and ghrehlin which plays a pivotal role in obesity \\
- Chronic lack of sleep decreases the production of serotonin, the happy hormone and thus leads to \\
- depression \\
- Higher levels of insulin which in turn promote fat storage and increase the risk for type II diabetes \\
Gastroesophageal reflux disorder is commonly associated with disturbed sleep
\end{tabular}

ventral periaqueductal gray (vPAG) contains Dopamine producing neurons (DA) that are active during wakefulness only. DA neurons in the $v$ PAG project to and receive input from cholinergic neurons in different areas in cerebral cortex. Loss of VPAG DA neurons promotes sleep. Thereby, DA agonists acting at D1, D2, and D3 receptors increase waking and decrease NREM and REM sleep. ${ }^{4}$

The homeostatic (the too much waking) signal is a reflection of fatigue and tiredness, and occurs when one is physically and mentally exhausted. However, this is not enough to generate sleep. Besides this, the circadian signal (time to sleep) is of equal importance. Suprachiasmatic nucleus helps maintain alertness by producing an alerting signal during the day and maintaining sleep by a reduced signal at night. Only when the homeostatic and circadian rhythm come together, does one fall asleep. It would be interesting to know that the homeostatic signal is linked with an increase in adenosine levels, and the effects of adenosine can be blocked by caffeine. Thus, coffee can overcome the homeostatic effects of sleepiness. However, it is ineffective against the circadian component of sleepiness.

\section{Sleep Architecture}

Sleep architecture is a term used to denote the structure of sleep. Sleep occurs in cycles, average length 1.5 hours, during which we go from light to deep sleep and back again. As we close our eyes, it takes 3-15 minutes to enter stage one. In the course of the night, we alternatively enter into two phases of sleep: NREM sleep (nonrapid eye movement) and the REM sleep (rapid eye movement).

\section{NREM Phase Sleep}

Based on electroencephalogram (EEG) findings, NREM sleep is divided into stage 1 to stage 3 . Despite previous literature showing 4 different stages, current data combines stage 3 and 4 as slow wave sleep. In normal young adults when he/ she falls to sleep EEG activity changes from alpha activity (18-30 Hz cycle/sec) to relatively low voltage mixed frequency pattern i.e. stage 1 which generally lasts for $1-10$ minutes. This is followed by $10-20$ minutes of stage 2 sleep, which has $\mathrm{K}$ complexes and sleep spindles. Slow wave sleep (Stages 3 and 4) where frequency is less than $2 \mathrm{~Hz}$ with high voltage activity of 75 $\mathrm{mv}$. Stage 3 lasts for few minutes only. Then stage 4 also called as low voltage sleep accounts for more than $50 \%$ EEG activity lasting for 20-40 minutes. Slow wave sleep stage of NREM is the most restorative of all.

\section{REM Phase Sleep}

REM sleep follows NREM sleep either in stage 1, stage 2, stage 3 or stage 4. REM sleep phase can further be divided into tonic REM and phasic REM depending upon absence or presence of short lived burst of eye movements. Over the sleeping hours from night to early morning the duration and frequency of REM phase increases and the quantum of slow wave sleep decreases. REM phase is also called "paradoxical sleep" as EEG resembles awake pattern. During REM phase, except for extraocular muscles and diaphragm, all other muscles in human body are atonic, thermoregulatory response to ambient temperature is absent, tidal volume and breathing frequency becomes variable, ventilator response to hypercarbia and hypoxia are reduced, and heart rate as well as blood pressure variability is noted. However, brain blood flow, oxygen consumption, glucose utilization stay similar to wakefulness. Dreaming and penile erection in males is a phenomenon seen in REM phase. REM sleep phase is vital for creativity.

Sleep deprivation is a relative concept. Small amounts of sleep loss (e.g. 1 hour per night over many nights) may have subtle cognitive repercussions, which may initially go unrecognized. Acute sleep loss results not only in loss of mental acuity and clarity but also affects cognitive functions. Chronic sleep deprivation has deeper ramifications (Table 2). The obvious signs of sleep deprivation are excessive sleepiness, yawning, irritability, and daytime fatigue. Deprivation of the deeper stages of sleep, bring about short and long-term consequences.

Frequent travellers in time zones who experience jetlag, and shift workers who have disturbed circadian rhythm patterns are even more prone to the above. Several cancers are commoner in night shift workers. Important is the fact that these sleep debts cannot be repaid and cannot be compensated for easily. Even catching up on sleep over weekends does not mitigate the debt. The most common cause of loss of sleep or insomnia is a change in your daily routine. For example, traveling, change in work hours, disruption of other behaviors (eating, exercise, leisure etc.), and relationship conflicts can all cause sleep problems.

\section{Sleep Hygiene}

Having understood what "Tinkering of Time of Sleep leads to", its equally important to understand how to ensure a good night's sleep for a productive day ahead. Early to bed and early to rise: the Lark versus the Owl, are always points of discussion. What is to be upheld is the fact that proximity to nature and following the 
natural homeostatic and circadian rhythms, leads to a refreshing sleep. Sleep hygiene is all about creating routines along with a conducive and pleasant environment at bedtime to promote sleep. Setting a schedule and a pre-bedtime routine, certainly helps. Sex naturally induces sleep. When people have sex, the body will release neuropeptides known as endorphins. Endorphins are the driving force behind many natural states of euphoria, like the runner's high, for instance.

\section{Do's}

- Go to bed at same time each day

- Get up from bed at same time each day.

- Regular exercise, preferably in the morning including stretching and aerobic exercise.

- Regular exposure to bright lights, especially in the late afternoon.

- Keep the bedroom dark, quiet and comfortable

- Use your bed only for sleep and sex.

- Take sleeping pills in the prescribed way only, i.e. 1 hour before bedtime or 10 hours before getting up.

- Use a relaxation exercise just before going to sleep. Muscle relaxation, imagery, massage, warm bath, etc.

- Keep your body warm while going to sleep

- Prefer foods which produce tryptophan like milk, oats, bananas, greens and some fruits are conducive to sleep at bedtime

\section{Don't}

- Exercise just before going to bed.

- Engaging in stimulating activity just before bed, such as playing a competitive game, watching an exciting program on television or movie, or having an important discussion with a loved one.

- Caffeine in the evening (coffee, many teas, chocolate, sodas, etc.)

- Use of blue light emitting electronic gadgets in bed

- Alcohol consumption to help you sleep.

- Go to bed too hungry or too full.

- Take over-the-counter sleeping pills, without your doctor's knowledge.

- Regular daytime naps

- Spending time in your bed struggling to get sleep more than 20-30 minutes.

- Commanding ones brain to forcefully sleep.

\section{Pregnancy and Sleep 5}

In pregnant women, sleep pattern changes from 1st to 3rd trimester. In 1st trimester, sleep time, daytime sleepiness and nocturnal awakening increases which later on with time normalizes. Slow wave sleep is comparatively reduced in 1st trimester and REM sleep is reduced in 3rd trimester. Sleep loss in pregnancy more than 7 hours leads to prolonged labour and a higher incidence of cesarean sections.

\section{Sleep and Perioperative Care}

Besides sleep in routine life, inadequate sleep due to obstructive sleep apnea (OSA) can create a whole lot of perioperative complications. Hence, assessment of sleep disordered breathing (SDB)/ OSA should be routine and mandatory for every operative fitness.
When suspected and an elective surgery is planned, sleep study should be performed. When an emergency surgery is planned, it is practical to use a CPAP machine pre and postoperatively. As a perioperative care, sedation should be avoided. For those who are already using their CPAP/BPAP, it is wise to continue using them. CPAP is contraindicated pre, intraoperative and postoperative in patients undergoing oropharyngeal and upper GI reconstructive surgery and skull based surgery to avoid aspiration. In mild cases postural therapy suffices.

American Society of Anesthesiology (ASA), the Society of Ambulatory Anesthesia (SAMBA) and the Society of Anaesthesia and Sleep Medicine (SASM) have published following recommendations: ${ }^{6}$

- OSA screening should be made part of standard preoperative assessment.

- Canceling or delaying surgery for a formal diagnosis (polysomnography) is not necessary unless, there is associated uncontrolled systemic disease.

- In planned surgeries, details of the sleep study and the recommended PAP pressures should be available.

- In patients with suspected/known/partially treated/untreated OSA who have uncontrolled systemic conditions (e.g. hypoventilation syndromes, severe pulmonary hypertension and resting hypoxemia in the absence of other cardiopulmonary disease) need preoperative cardiopulmonary optimization.

- Opioid Analgesics are to be avoided, and awake extubation is recommended for all OSA patients.

\section{Pearls of Wisdom}

- Sleep is an integral part of health for maintaining a good quality of life

- Always ensure a sleep architecture by following a routine daily

- Follow do's and don'ts for sleep hygiene in day-to-day life

- An SDB, especially OSA, is known to affect morbidity and mortality in perioperative period

- Pregnancy and OSA have an established association

\section{References}

1. Ohayon MM, Carskadon MA, et al. Meta-analysis of quantitative sleep parameters from childhood to old age in healthy individuals: developing normative sleep values across the human lifespan. Sleep 2004;27:1255-1273.

2. De Lecca L, Kilduff TS, et al. The hypocretins: hypo- thalamic specific peptides with neuroexcitatory activity. Proc Natl Acad Sci USA 1998;95:322-327.

3. Sakurai T. Orexins and Orexin receptors: a family of hypotha- lamic neuropeptides and $\mathrm{G}$ protein coupled receptors that regulate feeding behavior. Cell 1998;92:573-585.

4. LuJ, Jhou TC, et al. Identificationofwake-activedopami- nergic neurons in the ventral periaqueductal gray matter. J Neurosci 2006;26:193-202.

5. N Edwards, et al. Sleep disordered breathing and pregnancy.Thorax 2002;57:555-558.

6. American Society of Anesthesiologists Task Force on Perioperative Management of Patients with Obstructive Sleep Apnea. Practice guidelines for the perioperative management of patients with obstructive sleep apnea: An updated report by the American Society of Anesthesiologists Task Force on Perioperative Management of Patients with Obstructive Sleep Apnea. Anesthesiology 2014;120:268286. 